\title{
A Scoping Review of the Health Benefits of Nature-Based Physical Activity
}

\author{
Richard W. Christiana ${ }^{1,2}$, Gina M. Besenyi ${ }^{2,3}$, Jeanette Gustat ${ }^{2,4}$, Teresa H. Horton ${ }^{2,5}$, Teresa L. \\ Penbrooke ${ }^{2,6}$, and Courtney L. Schultz ${ }^{2,7}$ \\ ${ }^{1}$ Appalachian State University, Beaver College of Health Sciences, United States \\ ${ }^{2}$ Centers for Disease Control and Prevention, Physical Activity Policy Research and Evaluation Network, \\ Parks and Green Space Work Group, Atlanta, GA, United States \\ ${ }^{3}$ Kansas State University, College of Health and Human Sciences, United States \\ ${ }^{4}$ Tulane University, School of Public Health and Tropical Medicine, United States \\ ${ }^{5}$ Northwestern University, Department of Anthropology, United States \\ ${ }^{6}$ GP RED, Healthy Communities Research Group, Louisville, CO, United States \\ ${ }^{7}$ Health \& Technology Partners, LLC, Milwaukee, WI, United States
}

\begin{abstract}
The health benefits of physical activity and spending time in nature are well established. However, youths and adults in the United States are not participating in sufficient levels of physical activity and are not spending much time outdoors. Recently, the need for equitable access to nature for all populations has been receiving more public health attention, though a specific focus on nature-based physical activity has been limited. The purpose of this scoping review is to operationalize the health benefits of nature-based physical activity in order to provide guidance for collaborations to program administrators, advocates, and researchers. Peer-reviewed literature is found in PubMed, Medline, Web of Science, and Google Scholar as well as in published reviews of the literature. The literature is divided into three categories of: 1) amount and location of nature-based components and physical activity; 2) added health benefits of exposure to nature-based components and physical activity; and 3) nature-based components and physical activity effect on non-white, marginalized, and vulnerable populations. This review supports and encourages multiple strategies to increase nature-based physical activity as this provides even greater benefit to health and wellness than exposure to nature or physical activity alone. Although many of the physical and mental health benefits of nature and physical activity are well established, additional research is needed to better understand the relationship between exposure to nature and nature-based physical activity, which will require greater investment and support from funding agencies.
\end{abstract}

Keywords: green exercise, mental health, nature-based components, nature-based initiatives, nature-based physical activity, outdoor physical activity 


\section{Introduction}

The health benefits of physical activity (PA) are well established and include reduced risk of heart disease, stroke, hypertension, type II diabetes, certain types of cancer, and decreased symptoms of depression (Piercy et al., 2018). However, research also indicates that youths and adults are not engaging in sufficient PA to achieve these benefits. Recent estimates suggest that only $24-26 \%$ of children and adolescents are meeting the recommended aerobic guidelines of at least 60 minutes of moderate-tovigorous PA (MVPA) per day (Katzmarzyk et al., 2018) while only $53.3 \%$ of adults are meeting recommended aerobic guidelines of at least 150 minutes of MVPA per week (Schiller et al., 2018). Given the health benefits and low participation rates, encouraging PA has been a focus of public health efforts including promoting structured exercise, active transportation (e.g., walking or biking for transport), and leisure-time PA.

A large and increasing body of literature points to the salutogenic effects of spending time in nature regardless of whether that time includes passive (e.g., viewing landscapes) or physically active behaviors (e.g., walking or hiking) (Bratman et al., 2019; Frumkin et al., 2017; Hartig et al., 2014; Kondo et al., 2018; McDonald et al., 2018; Twohig-Bennett \& Jones, 2018). However, along with reduced PA levels, a decline has also occurred in the amount of time people spend outdoors engaging with nature. One cross-sectional study of over 2,000 children showed that between the years of 1981-82 and 2002-03 the amount of time children spent in unstructured outdoor activities declined from 100 minutes per week to 50 minutes per week (Hofferth \& Sandberg, 2001; Juster et al., 2004). Similarly, the Canadian Human Activity Pattern Survey (CHAPS) reports that in the 15 years between the first survey (CHAPS 1, early to mid-1990's) and second (CHAPS 2, 2010-11) the amount of time youths spent outdoors decreased by 24-35 minutes per day, depending on age group (Matz et al., 2014). With respect to adults, the National Human Activity Pattern Survey estimated that during the period of 1992-94, adults in the United States spent about 109 minutes a day outdoors (Klepeis et al., 2001). Few studies report on historical trends in time outside or how time outside is spent by adults, but changes in video screen use and sedentary time suggest that the amount of time adults spend inside is increasing (Beyer et al., 2018; Du et al., 2019; Livingston, 2019). This impression is supported by a national survey of approximately 5,500 adults in the United States who reported that during 2015-16, half spent fewer than 5 hours per week, or less than $10 \%$ of each day, outdoors in nature, excluding organized sports in a typical week (Kellert \& Case, 2018). It is important to note that rates of participation in outdoor recreation have increased markedly during the COVID-19 pandemic. According to the Physical Activity Council's annual participation survey, 2020 saw the highest rate of participation in outdoor recreation with $53 \%$ of Americans ages 6 and older recreating outdoors at least once (Outdoor Foundation, 2021). However, there is reason to believe that this increased participation could be temporary as only $20 \%$ reported participating in outdoor activities more than twice a week, continuing an existing downward trend prior to the pandemic, and a quarter of new participants in outdoor recreation indicated no desire to continue to participate in outdoor activities (Outdoor Foundation, 2021).

\section{Nature health benefits}

The foundational hypotheses for how engaging with nature improves health and well-being are derived from multiple fields of research, but with environmental psychology being the most common (Spencer \& Gee, 2009). These hypotheses include the Attention Restoration Theory (Kaplan \& Kaplan, 1989), the Stress Reduction Theory (Ulrich et al., 1991), and the Biophilia Hypothesis (Kellert \& Wilson, 1995). More recently, additional hypotheses propose that cultural learning and place attachment contribute to the salutogenic effects of nature (Beery et al., 2015; Joye \& De Block, 2011; Knez et al., 2018; Sampson, 2012). In brief, these hypotheses posit that engaging with nature improves mental and physical health via psychobiological pathways associated with changes in neuroendocrine, immune, and autonomic nervous system functions. In addition to these psychobiological pathways, evidence for more direct physiological pathways is increasing. For example, inhaling specific plant compounds (phytoncides) is associated with improved immune function (Kuo, 2015; Oh et al., 2017; Tsao et al., 2018). The presence of vegetation may also improve well-being by reducing exposure to air pollution or reducing urban heating (Dadvand et al., 2015; Donovan et al., 2013; Livesley et al., 2016; Yang et al., 2019). Exposure to nature also may influence the body's microbiome leading to changes in health status (Lowry et al., 2016; Prescott \& Logan, 2016). Finally, given the recent effects of the COVID-19 pandemic, research evaluating the effects of the natural and built environment on PA and the impacts of disease transmission are increasingly relevant (PinterWollman et al., 2018). Thus, this rich body of evidence provides the backdrop for exploring synergies between PA, the location where PA occurs, and the role of nature in public health.

\section{Nature-based components}

When evaluating spaces in communities, the language used in research and planning has often included different, and sometimes conflicting, terms around natural places, especially in different geographic areas (Layton, 2018). Table 1 provides definitions for key terms related to natural places to aid practitioners and researchers in working in this area. Recent research and professional practice in the United States has shifted to the use of the broad term "components" to describe the variety of places, lands, and facilities that are afforded to an individual (Layton, 2018). Components are defined as those things that individuals can visit and use in a community. These can include active spaces, such as ballfields, courts, picnic facilities, playgrounds, and trails. Modifiers can be safety, comfort and convenience amenities that support or enhance the overall experience of visiting the spaces, including such things as the availability of restrooms, drinking water, 
shade, seating and overall comfort, convenience, scenic quality, accessibility features for populations with disabilities, and lighting (Layton, 2018). Additionally, the effect of racial discrimination and racial profiling has been identified as a key element to the safety and use of these spaces by non-white, marginalized populations (Lee \& Scott, 2016).

Nature-based components are often called parks, green spaces, natural areas, conservation areas, forests, wateraccess, greenways, and a variety of other terms. Typically, these spaces have been defined as areas with predominant vegetative and/or geological features that reflect natural processes (e.g., trees, prairies, grasses) (Centers for Disease Control and Prevention, 2009; Frumkin et al., 2017; Hartig et al., 2014; Lachowycz \& Jones, 2013; Taylor \& Hochuli, 2017; World Health Organization, 2016). However, while much emphasis is placed on vegetation and greenness, this definition and the concept of restorative nature suggest that other types of natural environments such as water and desert environments may also provide benefits (De Vries, 2019; Kaplan \& Kaplan, 1989; Kelly, 2018).

For simplicity, and because the body of literature reviewed for this paper emphasizes areas with trees and vegetation, in this commentary we will use the term components (typically meaning nature-based). The term green exercise has been applied to mean activity that is undertaken in locations that meet the definition of naturebased components or green space given above (Fraser et al., 2019; Olafsdottir et al., 2017; Pretty et al., 2003). We refer to nature-based PA rather than green exercise.

\begin{tabular}{|c|c|c|}
\hline Term & Definition & Reference(s) \\
\hline Blue Space & $\begin{array}{l}\text { All visible surface water in a space; including } \\
\text { oceans, lakes, rivers, streams, ponds, and other } \\
\text { such waterscapes. }\end{array}$ & $\begin{array}{l}\text { (Völker \& Kistemann, } \\
\text { 2011) }\end{array}$ \\
\hline Brown Space & $\begin{array}{l}\text { Areas where the space is dominated by soil } \\
\text { type, rocks, and green is less prominent such as } \\
\text { arid regions including desert landscapes. }\end{array}$ & (Nazif-Munoz et al., 2020) \\
\hline Gray Space & $\begin{array}{l}\text { Areas dominated by concrete, buildings, and } \\
\text { other impervious surfaces typically } \\
\text { characteristic of human-constructed } \\
\text { environments. }\end{array}$ & (Nazif-Munoz et al., 2020) \\
\hline Green Exercise & $\begin{array}{l}\text { Physical activity undertaken in both urban and } \\
\text { nonurban natural environments. }\end{array}$ & (Pretty et al., 2005) \\
\hline Green Space & $\begin{array}{l}\text { Areas dominated by natural and/or planted } \\
\text { vegetation such as grass, plants, or trees. }\end{array}$ & $\begin{array}{l}\text { (Klompmaker et al., 2018; } \\
\text { Taylor \& Hochuli, 2017) }\end{array}$ \\
\hline Natural Environments & $\begin{array}{l}\text { Landscapes dominated by blue space, green } \\
\text { space, and/or brown space that contain flora } \\
\text { and fauna that are minimally influenced by } \\
\text { humans. }\end{array}$ & $\begin{array}{l}\text { (Johnson et al., 1997; } \\
\text { McIsaac \& Brün, 1999) }\end{array}$ \\
\hline Nature-based Components & $\begin{array}{l}\text { Often called parks, green spaces, natural areas, } \\
\text { conservation areas, forests, water-access, } \\
\text { greenways, and a variety of other terms. } \\
\text { Typically, these spaces have been defined as } \\
\text { areas with predominant vegetative and/or } \\
\text { geological features that reflect natural } \\
\text { processes (e.g., trees, prairies, grasses). }\end{array}$ & $\begin{array}{l}\text { (Frumkin et al., 2017; } \\
\text { Lachowycz \& Jones, 2013; } \\
\text { Layton, 2018; Taylor \& } \\
\text { Hochuli, 2017; World } \\
\text { Health Organization, 2016) }\end{array}$ \\
\hline Nature-based Interventions & $\begin{array}{l}\text { Programs, activities or strategies that aim to } \\
\text { engage people in nature-based experiences } \\
\text { with the specific goal of achieving improved } \\
\text { health and well-being }\end{array}$ & (Shanahan et al., 2019) \\
\hline Nature-based Therapeutic Interventions & $\begin{array}{l}\text { Use of a natural area, specifically designed or } \\
\text { chosen, for a therapeutic intervention. }\end{array}$ & (Stigsdotter et al., 2011) \\
\hline
\end{tabular}

\section{Role of public health}

The public health community is often torn between the desire to act and the desire for more evidence. Nowhere is this more apparent than with the rising interest in the role of nature-based components and PA as a means to improve public health. For example, in 2013 the American Public Health Association (APHA), the largest organization of public health professionals in the United States, promoted a policy statement (APHA PS20137) highlighting the relationship between time spent outdoors and PA and recommended 11 action steps to increase access to nature to improve public health (Chawla \& Litt, 2013). The existence of this policy statement is a direct and profound declaration that it is in the interest of public health for 
people to spend time in nature, regardless of whether that time is spent in PA or not.

Along these lines, in an article in Environmental Health Perspectives, Frumkin and colleagues (2017) describe the gaps in our knowledge about how exposure to nature improves health and propose a research agenda to address these key gaps in knowledge including the following: 1) the dosage of nature needed for significant health benefit, 2) the biomarkers of exposure to nature, 3 ) clarity on whether nature-based PA provides greater benefits than the equivalent PA in a non-nature based setting, and 4) the best strategies and approaches to promote exposure to nature within populations; including understanding what components of nature are salutogenic. Additionally, two more recent review articles state that the number of papers providing evidence for the beneficial effects of nature to specific health conditions is too low and the diversity of measures used too wide to make generalizable statements (Kondo et al., 2018; Twohig-Bennett \& Jones, 2018).

The primary goal of this scoping review is to provide a foundation for those seeking to understand the breadth of information on the positive associations between exposure to nature-based components, PA, and health outcomes. We also provide guidance to program administrators, advocates, and researchers that will facilitate collaborations, promote evaluation and research programs, and inform interventions in the interest of identifying existing evidence that supports actionable programs and environment improvements.

\section{Methods}

The eleven broad categories of evidence on the connections between nature and health presented in the 2013 APHA policy statement served as the starting point for the literature review (Chawla \& Litt, 2013). The current literature review focused on the connection between naturebased PA and specific positive health outcomes. Research literature focusing on nature-based PA in each of these eleven broad categories was searched in PubMed, Medline, Web of Science, and Google Scholar. In addition, original research was found in previously published reviews of the literature. The authors, representing a diverse range of academic and professional disciplines (biological anthropology, public health, kinesiology, and parks and recreation planners and practitioners) brought together through the CDC-organized Physical Activity Policy Research and Evaluation Network (PAPREN), met monthly to discuss the literature found. The literature was divided into three categories of: 1) Amount and location of nature-based components and PA; 2) Added health benefits of exposure to nature-based components and PA; and 3) Nature-based components and PA effect on non-white, marginalized, and vulnerable populations. Within each of these categories, subcategories were established to help summarize the breadth of evidence in meaningful ways for a variety of stakeholders. When exploring the literature for such evidence, descriptions of nature exposure included time spent outdoors or in a variety of environments commonly labeled as natural, green, blue, or brown environments.

It should be noted that this is not meant to be a systematic or comprehensive review of the literature. Rather, our goal was to provide a foundation for those seeking to understand the breadth of information on the positive associations between exposure to nature-based components and PA and health outcomes.

\section{Results}

To facilitate reading clarity, citations have been consolidated into tables associated with each section. Citations have been included in the text only when they are not directly associated with material contained in a table.

\section{Amount and location of nature-based components and PA}

Research has examined the relationship between the amount, proximity, and quality of neighborhood components on PA-related behavior across the lifespan. Amount of the components refers to the total number of available components, often described through amount of acreage or objective vegetative analyses that is within a "reasonable" distance (to walk, bike, or drive to) from a person's home. Location or proximity refers to the distance of the nearest component. Quality refers to the modifiers features, amenities, and facilities of nearby components, such as maintained equipment, lighting, water fountains, bathrooms, parking, and trees for shade, biodiversity, and aesthetics. The literature reviewed below on the amount and location of nature-based components and PA is summarized in Table 2.

\section{Children and Adolescents}

Among children ( $<10$ years of age) and adolescents (1018 years of age), the amount of green space in the neighborhood is positively associated with increased MVPA. Among children and adolescent girls, closer proximity to green space is associated with increased MVPA. Several studies have found that in addition to adolescents having access to green space, spaces with higher quality facilities are associated with more MVPA, less sedentary behavior, and greater use of the green space.

\section{Adults}

Among adults (18-65 years of age), having more access to components is associated with increased MVPA and increased walking. Research shows that closer proximity to components is associated with increased MVPA, increased use, and increased walking. Increased MVPA and increased walking is also associated with the availability of both components and with quality modifying features (i.e., safe, aesthetically pleasing, has trees). Furthermore, one study found that perceived quality was a better predictor of visit frequency than objective measures of quantity of components (Flowers et al., 2016). Lastly, components with higher biodiversity as well as high quality modifiers 
provided greater levels of psychological restoration than components with lower biodiversity.

\section{Older Adults}

Among older adults ( $>65$ years of age) specifically, the amount of neighborhood components is related to higher levels of MVPA and increased sports-related activity. In addition, modifying features and amenities of components, as mentioned previously, were related to increased leisuretime PA while quality of components has been related to less decline in walking among older adults.

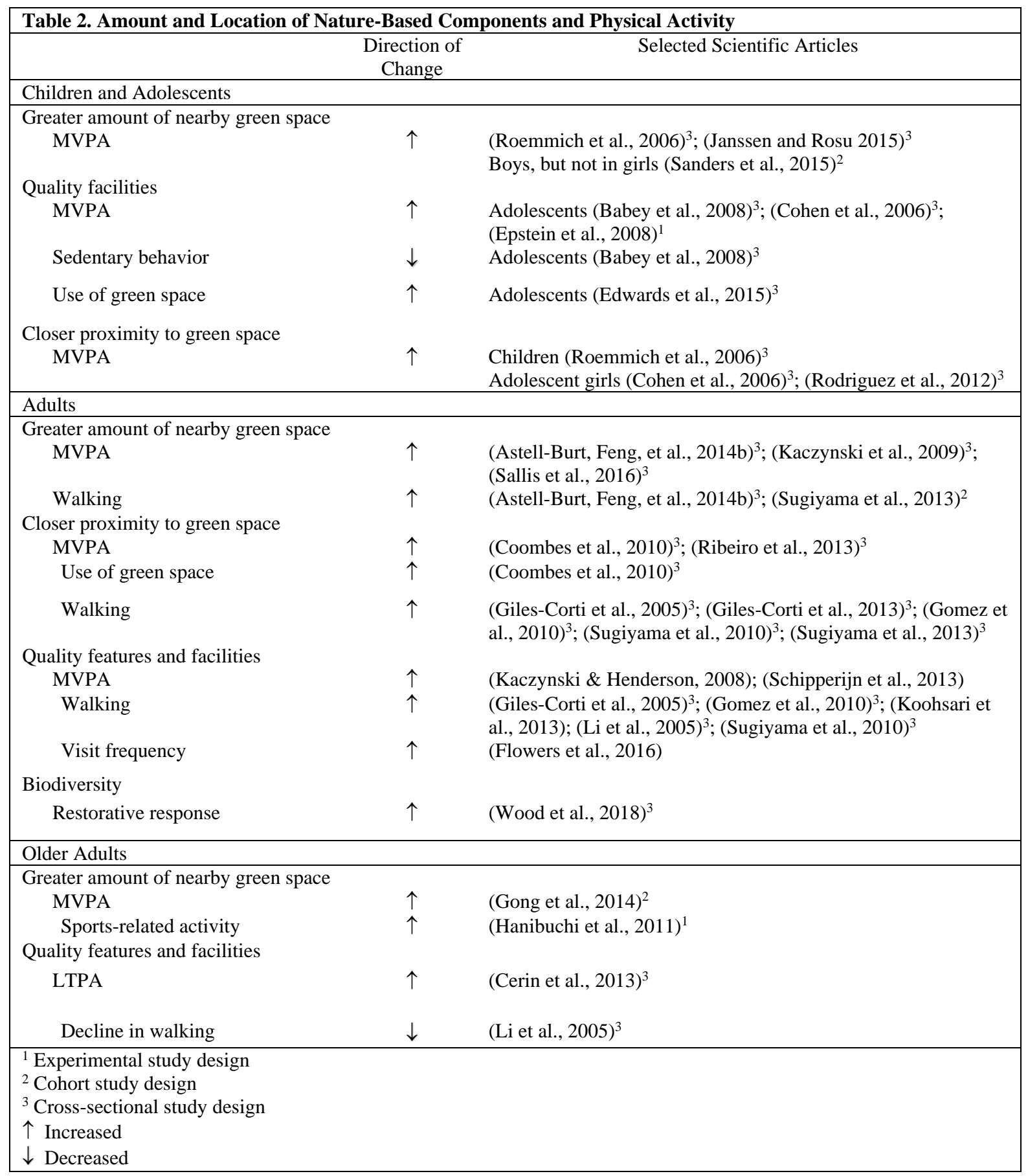




\section{Added health benefits of exposure to nature-based components and PA}

Beyond the benefits of PA, numerous benefits to physiological, mental, cognitive, and social health have been associated with both exposure to nature and naturebased PA. These are summarized in Table 3 .

\section{Physiological}

A large number of anthropometric, biochemical, and neural outcomes have been used to assess the impact of engaging with nature on physiology and health. Nature and nature-based PA is associated with decreased heart rate, blood pressure, cholesterol, body mass index (BMI), and improved heart rate variability. Biochemical responses include positive impacts on enzymes such as alpha-amylase which aids glucose absorption; hormones such as cortisol (linked with stress and metabolic regulation) and dehydroepiandrosterone sulfate (linked with sex drive, osteoporosis and dementia); the immune system (e.g., interleukin-6 (IL-6), natural killer cells); and neurotransmitters such as noradrenaline, which mobilizes the brain and body for action, and dopamine, which plays a role in the motivational element of reward-motivated behavior. Greater parasympathetic and lower sympathetic nerve activity and improved sleep and circadian rhythms resulting from time spent outdoors has been found to further support nature's restful and restorative effects. As might be expected given the many positive impacts on physiological parameters, exposure to nature is associated with reduced incidence of stroke, hypertension, dyslipidemia, asthma, and coronary heart disease as well as risk of obesity, diabetes, preterm birth, small size for gestational age, cardiovascular mortality, and all-cause mortality. As noted in multiple meta-analyses, the diversity of measures and outcomes makes it difficult to arrive at generalizable trends or tailored recommendations for specific conditions. Therefore, more rigorous and coordinated research efforts are needed to better establish the physiological benefits of nature and nature-based PA (Bowler et al., 2010; Lachowycz \& Jones, 2011; Thompson Coon et al., 2011; Twohig-Bennett \& Jones, 2018).

\section{Psychological/Emotional}

Exposure to nature-based components and PA has long been associated with improved mental and emotional well- being, including increases in positive engagement, revitalization, relaxation, self-esteem, body image, energy, affective response, self-reported health and health-related quality of life. Likewise, exposure to nature is associated with reductions in negative symptoms and outcomes such as stress, anxiety, depression, and fatigue (World Health Organization, 2016). Exposure to nature has been shown to be an effective treatment for anxiety by providing a spectrum of sensory stimulations that focus attention and allow emotional processing to be external instead of internal (Detweiler et al., 2018).

\section{Cognitive Function and Experience}

Multiple mental abilities such as attention, learning, thinking, reasoning, remembering, problem solving, and decision-making are associated with exposure to naturebased components and PA. Experimental studies have shown that spending time in nature improves memory function, direct attention, increased neural activity associated with deep meditative states and daydreaming, improved child development, greater intelligence and academic performance, and lower levels of arousal and frustration. Further, nature exposure can influence how we make decisions about PA. For example, those who spend more time outdoors and interact more with nature report lower perceived effort for exercise and have greater frequency and duration of MVPA. Some studies also show that outdoor environments can influence preferred activities and types of play as well as engage less fit populations.

\section{Social Relationships}

Our social relationships play a major role in our overall health. Cross-sectional studies indicate that spending time in nature fosters social capital and social support that a person receives from others. This may be because time spent in nature provides opportunities and activities for socialization and formation of social networks. Nature exposure is also associated with greater social cohesion, a key aspect of the Healthy People 2020 Social and Community context domain, referring to the "strength of relationships and the sense of solidarity among community members" (US Department of Health and Human Services, 2010). This area of literature also points to the role nature and nature-based PA can have on social mobility and factors that may impact income/wealth accumulation such as health care spending.

\begin{tabular}{|c|c|c|}
\hline & $\begin{array}{l}\text { Direction } \\
\text { of Change }\end{array}$ & Selected Scientific Articles \\
\hline \multicolumn{3}{|c|}{ Physiological (cardiovascular and metabolic parameters) } \\
\hline Blood pressure & $\downarrow$ & $\begin{array}{l}\left.\text { (Park et al., 2010) })^{1} \text {; (Twohig-Bennett \& Jones, 2018) }\right)^{2} \text {; (Li et al., } \\
2011)^{1} \text {; (Yang, Markevych, Bloom, et al., 2019) }\end{array}$ \\
\hline Diastolic blood pressure & $\downarrow$ & $\left(\right.$ Twohig-Bennett \& Jones, 2018) ${ }^{2}$ \\
\hline Heart rate & $\downarrow$ & $\left(\right.$ Park et al., 2010) ${ }^{1} ;\left(\right.$ Twohig-Bennett \& Jones, 2018) ${ }^{2}$ \\
\hline Heart rate variability & $\uparrow$ & $(\text { Blum et al., 2019) })^{1} ;(\text { de Brito et al., 2020) })^{1}$ \\
\hline Low frequency & $\downarrow$ & $(\text { Twohig-Bennett \& Jones, 2018) })^{2} ;\left(\right.$ Park et al., 2017) ${ }^{3}$ \\
\hline
\end{tabular}




\begin{tabular}{|c|c|c|}
\hline $\begin{array}{l}\text { High frequency } \\
\text { Nighttime }\end{array}$ & $\begin{array}{l}\uparrow \\
\uparrow\end{array}$ & $\begin{array}{l}\text { (Twohig-Bennett \& Jones, 2018) }{ }^{2} \\
(\text { Gladwell et al., 2016) }\end{array}$ \\
\hline Body mass index (BMI) & $\downarrow$ & $\begin{array}{l}\text { (Veitch et al., 2016) }{ }^{4} \text {; (Tilt et al., 2007) }{ }^{4} \text {; (Astell-Burt, Feng, et } \\
\text { al., 2014a) }{ }^{4} \text { (Li \& Ghosh, 2018) }{ }^{4} \text {; (O'Callaghan-Gordo et al., } \\
2020)^{4}\end{array}$ \\
\hline \multicolumn{3}{|l|}{ Sleep/circadian rhythms } \\
\hline $\begin{array}{l}\text { Align internal rhythms with natural } \\
\text { sunrise and sunset. }\end{array}$ & $\uparrow$ & (Wright et al., 2013) ${ }^{1}$ \\
\hline Sleep duration & $\uparrow$ & $\begin{array}{l}\text { (Shin et al., 2020) })^{2} ;(\text { Johnson et al., 2018) })^{4} ;(\text { Astell-Burt \& } \\
\text { Feng, 2020a })^{4} ;(\text { Wright et al., 2013) })^{1}\end{array}$ \\
\hline Sleep quality & $\uparrow$ & $(\text { Shin et al., 2020 })^{2} ;(\text { Pasanen et al., 2014) })^{4}\left(\right.$ Xie et al., 2020) ${ }^{4}$ \\
\hline HDL cholesterol & $\downarrow$ & (Twohig-Bennett \& Jones, 2018) ${ }^{2} ;($ Fan et al., 2020) \\
\hline Markers of oxidative stress & $\downarrow$ & (Mao, Lan, et al., 2012) ${ }^{1}$; (Mao, Cao, et al., 2012) ${ }^{1}$ \\
\hline Alpha-amylase & $\downarrow$ & $(\text { Egorov et al., 2017) })^{4}$; (Hunter et al., 2019) ${ }^{3}$ \\
\hline Cortisol & $\downarrow$ & $\begin{array}{l}\text { (Park et al., 2010) })^{1} ;(\text { Egorov et al., 2017) })^{4} \text {; (Hunter et al., } \\
\text { 2019) }{ }^{3} \text {; (Honold et al., 2016) }{ }^{4} ;(\text { Gidlow et al., 2016) } \\
\text { (Twohig-Bennett \& Jones, 2018) }^{2}\end{array}$ \\
\hline Adiponectin & $\uparrow$ & $(\text { Li et al., 2011) })^{1} ;(\text { Li et al., 2016) })^{1}$ \\
\hline Dehydroepiandrosterone sulfate & $\uparrow$ & $\left(\right.$ Li et al., 2011) ${ }^{1}$ \\
\hline $\begin{array}{l}\text { Pro-inflammatory cytokines (e.g., IL-6; } \\
\text { TNF- } \alpha \text { ) }\end{array}$ & $\downarrow$ & $\begin{array}{l}\text { (Mao, Lan, et al., 2012)후 (Mao, Cao, et al., 2012) }{ }^{1} ;(\text { Oh et al., } \\
2017)^{2}\end{array}$ \\
\hline $\begin{array}{l}\text { Anti-inflammatory cytokines (e.g., IL- } \\
\text { 8) }\end{array}$ & $\uparrow$ & (Egorov et al., 2017) $^{4}$ \\
\hline Natural Killer Cells & $\uparrow$ & $\left(\right.$ Li et al., 2007) ${ }^{1} ;(\text { Li et al., 2009) })^{1} ;\left(\right.$ Tsao et al., 2018) ${ }^{1}$ \\
\hline Allostatic Load & $\downarrow$ & (Egorov et al., 2017) $^{4}$; (Ribeiro et al., 2019) ${ }^{4}$ \\
\hline $\begin{array}{l}\text { Parasympathetic nerve activity } \\
\text { Heart rate variability }\end{array}$ & $\uparrow$ & $\begin{array}{l}\left.\text { (Farrow \& Washburn, 2019) })^{2} \text {; (Park et al., 2010) }\right)^{1} \text {; (Park et al., } \\
2017)^{1} \text {; (Lee et al., 2014) }{ }^{3} \text {; (Song et al., 2019) }{ }^{1}\end{array}$ \\
\hline \multicolumn{3}{|l|}{ Sympathetic nerve activity } \\
\hline Heart rate variability & $\downarrow$ & $\begin{array}{l}\left.(\text { Farrow \& Washburn, 2019) })^{2} \text {; (Park et al., 2010) }\right)^{1} \text {; (Park et al., } \\
\left.\text { 2017) })^{1} \text {; (Lee et al., 2014) }\right)^{3} \text {; (Song et al., 2019) }\end{array}$ \\
\hline $\begin{array}{l}\text { Urine adrenaline/noradrenaline/ } \\
\text { dopamine }\end{array}$ & $\downarrow$ & $\begin{array}{l}(\mathrm{Li} \text { et al., 2008) })^{1} ;(\mathrm{Li}, 2010)^{1} ;(\mathrm{Li} \text { et al., 2011 })^{1} ;(\mathrm{Li} \text { et al., } \\
2016)^{1}\end{array}$ \\
\hline \multicolumn{3}{|l|}{ Neurotransmitters } \\
\hline Noradrenaline/adrenalin & $\downarrow$ & (Li et al., 2011) ${ }^{1}$; (Egorov et al., 2017) \\
\hline Dopamine & $\downarrow$ & $(\text { Li et al., 2011 })^{1} ;(\text { Li et al., 2016 })^{1} ;(\text { Egorov et al., 2017 })^{4}$ \\
\hline \multicolumn{3}{|l|}{ Disease Prevalence/Risk } \\
\hline Asthma & $\downarrow$ & $\left(\right.$ Twohig-Bennett \& Jones, 2018) ${ }^{2}$; (Lovasi et al., 2008) ${ }^{4}$ \\
\hline Hypertension & $\downarrow$ & $\left(\right.$ Twohig-Bennett \& Jones, 2018) ${ }^{2}$ \\
\hline Dyslipidemia & $\downarrow$ & $\left(\right.$ Twohig-Bennett \& Jones, 2018) ${ }^{2}$ \\
\hline Obesity/metabolic syndrome & $\downarrow$ & 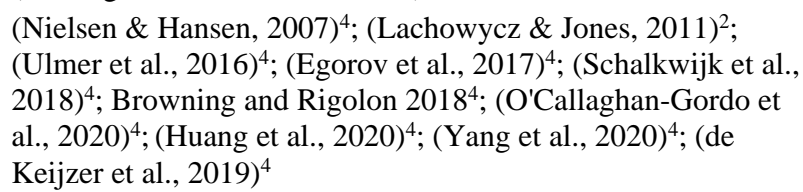 \\
\hline Stroke & $\downarrow$ & $\left(\right.$ Twohig-Bennett \& Jones, 2018) ${ }^{2}$; (Orioli et al., 2019) ${ }^{4}$ \\
\hline Coronary heart disease & $\downarrow$ & $\left(\right.$ Twohig-Bennett \& Jones, 2018) ${ }^{2}$; (Wang et al., 2019) ${ }^{4}$ \\
\hline Diabetes type II/gestational & $\downarrow$ & $\begin{array}{l}\text { (Twohig-Bennett \& Jones, 2018) }{ }^{2} \text {; (Bodicoat et al., 2014) } \\
(\text { Ulmer et al., 2016) } \\
\text { Markevych, (Egorov et al., 2017) }{ }^{4} \text {; (Yang, } \\
\text { 2014b) }{ }^{4} ;(\text { Qu et al., 2020) })^{4}\end{array}$ \\
\hline $\begin{array}{l}\text { Pre-term birth/small size for } \\
\text { gestational age }\end{array}$ & $\downarrow$ & (Twohig-Bennett \& Jones, 2018) ${ }^{2}$ \\
\hline Cardiovascular mortality & $\downarrow$ & $\left(\right.$ Twohig-Bennett \& Jones, 2018) ${ }^{2}$; (Yitshak-Sade et al., 2019) \\
\hline All-cause mortality & $\downarrow$ & 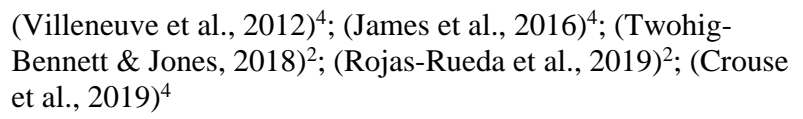 \\
\hline
\end{tabular}




\begin{tabular}{|c|c|c|}
\hline Psychological/Emotional Parameters & & \\
\hline $\begin{array}{l}\text { Well-being } \\
\text { Positive engagement } \\
\text { Revitalization } \\
\text { Tension } \\
\text { Confusion } \\
\text { Anger } \\
\end{array}$ & $\uparrow$ & 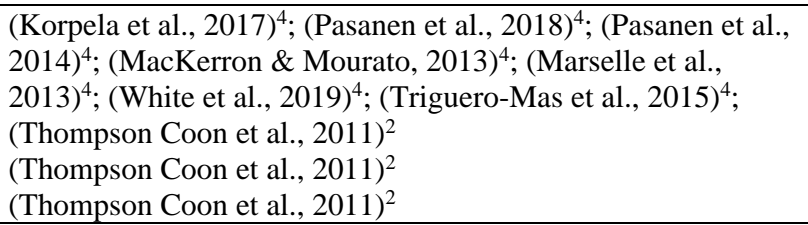 \\
\hline Risk of Poor Mental Health & $\downarrow$ & (Mitchell et al., 2013) $3^{4}$ \\
\hline $\begin{array}{l}\text { Emotional Experience } \\
\text { Relaxation } \\
\text { Frustration }\end{array}$ & $\begin{array}{l}\uparrow \\
\downarrow\end{array}$ & $\left(\right.$ Aspinall et al., 2015) ${ }^{3}$ \\
\hline $\begin{array}{l}\text { Self-esteem } \\
\text { Body image/appreciation }\end{array}$ & $\uparrow$ & $\begin{array}{l}\text { (Barton et al., 2012)3; (Barton \& Pretty, 2010) }{ }^{2} \text {; (Swami et al., } \\
2016)^{4} \\
(\text { Swami et al., 2019) }\end{array}$ \\
\hline Stress & $\downarrow$ & \\
\hline In children & $\downarrow$ & $\left(\right.$ Razani et al., 2019) ${ }^{1}$ \\
\hline In adults & $\downarrow$ & $\left(\right.$ Kondo et al., 2018) ${ }^{4}$; (Marselle et al., 2013) ${ }^{4}$; (Nielsen \& \\
\hline Stress related illnesses & $\downarrow$ & $\begin{array}{l}\left.\text { Hansen, 2007) })^{4} \text { (Largo-Wight et al., 2011 }\right)^{4} ; \text { (Wooller et al., } \\
2018)^{1} ;\left(\text { Corazon et al., 2018) }{ }^{4} ;(\text { Grahn \& Stigsdotter, 2003) }\right.\end{array}$ \\
\hline Risk of Psychological Distress & $\downarrow$ & (Francis et al., 2012) $^{4}$; (Astell-Burt \& Feng, 2019) ${ }^{4}$ \\
\hline Anxiety & $\downarrow$ & $\begin{array}{l}(\text { Niedermeier et al., 2017) })^{1} ;\left(\text { Bratman et al., 2015) }{ }^{1} ;(\text { Mackay \& }\right. \\
\text { Neill, 2010) }\end{array}$ \\
\hline Depression & $\downarrow$ & $\begin{array}{l}\text { (Thompson Coon et al., 2011)2; (Egorov et al., 2017) } \\
(\text { Irandoust \& Taheri, 2017) }\end{array}$ \\
\hline Rumination & $\downarrow$ & $\left(\right.$ Bratman et al., 2015) ${ }^{3}$ \\
\hline Child resilience & $\uparrow$ & (Razani et al., 2019) $^{1}$ \\
\hline Affective Response & $\uparrow$ & $\left(\right.$ Niedermeier et al., 2017) ${ }^{1}$ \\
\hline Affective valence & $\uparrow$ & $\left(\right.$ Bratman et al., 2015) ${ }^{1}$ \\
\hline Activation & $\uparrow$ & $\left(\right.$ Niedermeier et al., 2017) ${ }^{1}$ \\
\hline Elation & $\uparrow$ & $\left(\right.$ Niedermeier et al., 2017) ${ }^{1}$ \\
\hline Calmness & $\uparrow \downarrow$ & $\left(\right.$ Niedermeier et al., 2017) ${ }^{1}$ \\
\hline Negative affect & $\downarrow$ & $\begin{array}{l}\text { (Bowler et al., 2010) })^{2} ;(\text { Thompson Coon et al., 2011) } \\
\text { (Marselle et al., 2013) })^{4} \text { (Bratman et al., 2015) }\end{array}$ \\
\hline Mood & $\uparrow$ & $\begin{array}{l}(\text { Barton et al., 2012) })^{3} ;\left(\text { Gidlow et al., 2016) }{ }^{3} \text {; (Barton \& Pretty, }\right. \\
2010)^{2} \text {; (Wooller et al., 2018) }\end{array}$ \\
\hline $\begin{array}{l}\text { Vitality } \\
\text { Fatigue }\end{array}$ & $\uparrow \leftrightarrow$ & 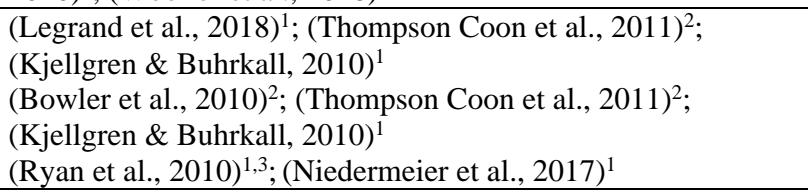 \\
\hline $\begin{array}{l}\text { Self-reported good health } \\
\text { Health-related Quality of Life } \\
\text { Poor Health }\end{array}$ & $\begin{array}{l}\uparrow \\
\downarrow\end{array}$ & 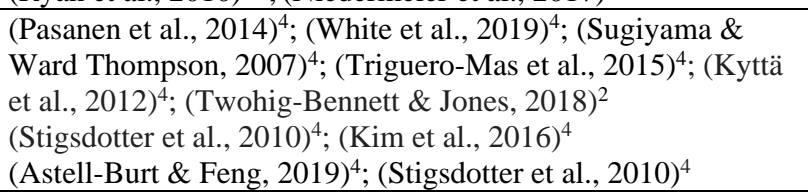 \\
\hline \multicolumn{3}{|l|}{$\begin{array}{l}\text { Cognitive Function } \\
\text { Cognition \& Experience Parameters }\end{array}$} \\
\hline Memory & $\uparrow$ & $\begin{array}{l}\left(\text { Gidlow et al., 2016) }{ }^{3} \text {; (Flouri et al., 2019) }{ }^{4} \text {; (Astell-Burt \& }\right. \\
\text { Feng, 2020b) }\end{array}$ \\
\hline Direct Attention & $\uparrow$ & $\left(\right.$ Bowler et al., 2010) ${ }^{2} ;($ Rogerson et al., 2016) \\
\hline Social Interaction Time & $\uparrow$ & $\begin{array}{l}\text { (Rogerson et al., 2016) }{ }^{3} \text {; (Aram et al., 2019) }{ }^{4} \text {; (Rasidi et al., } \\
2012)^{4}\end{array}$ \\
\hline Restoration Experience & $\uparrow$ & $\begin{array}{l}\text { (Gidlow et al., 2016) }{ }^{3} \text {; (Pasanen et al., 2018) } \\
2018)^{3} \text {; (Bailey et al., }\end{array}$ \\
\hline Healthy Child Development & $\uparrow$ & $(\text { Gill, 2014) })^{2}$ \\
\hline Intelligence/Academic Performance & $\uparrow$ & $\begin{array}{l}\text { (Browning \& Rigolon, 2019b })^{2} ;(\text { Bijnens et al., 2020) })^{4} \text {; Li et } \\
\text { al., 2019) }{ }^{4} \text { (Kuo et al., 2018) }\end{array}$ \\
\hline $\begin{array}{l}\text { Perception of Required Effort for } \\
\text { Exercise }\end{array}$ & $\begin{array}{c}\uparrow \downarrow \\
\downarrow\end{array}$ & $\begin{array}{l}(\text { Gladwell et al., 2013) } \\
(\text { Lahart et al., 2019) }\end{array}$ \\
\hline
\end{tabular}




\begin{tabular}{|c|c|c|}
\hline Nature Engagement & $\uparrow$ & $\left(\right.$ Han \& Wang, 2018) ${ }^{3}$ \\
\hline Frequency of Exercise & $\uparrow$ & $\left(\right.$ Hug et al., 2009) ${ }^{4}$ \\
\hline $\begin{array}{l}\text { Engagement in and Duration of } \\
\text { Moderate-to-Vigorous Physical } \\
\text { Activity }\end{array}$ & $\uparrow$ & $\begin{array}{l}\text { (Beyer et al., 2018) }{ }^{4} ;\left(\text { Wood et al., 2014) }{ }^{3} ;(\text { Byrka \& Ryczko, }\right. \\
2018)^{4} ;(\text { Cleland et al., 2008) }\end{array}$ \\
\hline Play Styles (Children) & $\uparrow$ & $\left(\right.$ Gill, 2014) ${ }^{2} ;\left(\right.$ Sandseter et al., 2020) ${ }^{4}$ \\
\hline \multicolumn{3}{|l|}{ Social Parameters } \\
\hline $\begin{array}{l}\text { Socialization Opportunities and } \\
\text { Activities }\end{array}$ & $\uparrow$ & $($ Fan et al., 2011) \\
\hline Social Support & $\uparrow$ & $($ Fan et al., 2011) \\
\hline Social Capital & $\uparrow$ & $(\text { Holtan et al., 2015) })^{4}($ Maas et al., 2009) \\
\hline Social Cohesion & $\uparrow$ & $\begin{array}{l}\left.\text { (Ruijsbroek et al., 2017) }{ }^{4} \text {; (de Vries et al., 2013) }\right)^{4} \text {; (Sugiyama } \\
\text { \& Ward Thompson, 2007) }{ }^{4} \text {; (Liu et al., 2020) }\end{array}$ \\
\hline Social Mobility & $\uparrow$ & (Browning \& Rigolon, 2019a) $^{4}$ \\
\hline Health Care Spending & $\downarrow$ & $\left(\right.$ Becker et al., 2019) ${ }^{4}$ \\
\hline $\begin{array}{l}{ }^{1} \text { Experimental study design } \\
{ }^{2} \text { Literature review or meta-analy } \\
{ }^{3} \text { Quasi experimental study desig } \\
{ }^{4} \text { Non-experimental/observationa } \\
\uparrow \text { Increased } \\
\downarrow \text { Decreased } \\
\uparrow \downarrow \text { Conflicting results } \\
\leftrightarrow \text { No change }\end{array}$ & & \\
\hline
\end{tabular}

Nature-based components and physical activity effect on non-white, marginalized, and vulnerable populations

Typically, low-income and racial/ethnic minority individuals and communities experience disparities in access to facilities and resources compared to higher income and non-Hispanic white individuals and communities. This is also reflected in that opportunities for PA are often not equal across all segments of society. Similarly, there are disparities in public policies associated with parks and recreation management (Spangler \& Caldwell, 2007). The literature reviewed is summarized in Table 4.

\section{Environmental Justice}

According to Bullard (2021), “environmental justice embraces the principle that all people and communities have a right to equal protection and equal enforcement of environmental laws and regulations... Reducing environmental, health, economic and racial disparities is a major priority of the Environmental Justice Movement." While the concept of environmental justice is broadly concerned with unwanted land use such as landfills, air and water pollution, commercial operations as well as tribal programs and policies, it has implications for components related to limiting nature exposure for low-income and minority groups in terms of urban design, access to and quality of outdoor recreation. Disparities in environmental justice are associated with disparities in outdoor PA opportunities and resources. For example, component quality has been found to vary by neighborhood race and income differences. These are likely related to historical and current disparities in policies, funding, and investment for various components in different neighborhoods and communities. One example is the continued effect of "redlining" or past discriminatory policies that restricted home ownership among racial minorities that resulted in various social and environmental issues still present today (Locke et al., 2021). These include urban neighborhoods with a high percentage of racial minorities having fewer nature-based components such as parks, green space, and trees for shade. Policies can help to address these disparities in public parks and recreation services. Calls have been made for public health and parks and recreation researchers and practitioners to collaborate on these topics.

\section{Non-White, Marginalized, and Vulnerable Populations}

A related issue has to do with Non-white marginalized and vulnerable populations' perceptions that natural spaces are controlled by and for dominant economic and racial groups (Finney, 2014). This may result in fear or less desire to use natural spaces as they may be seen as not welcoming or not for the desired uses of those groups. However, this concept may be associated with specific cultures and geographies (Hagerhall, 2018). Generally, marginalized and vulnerable groups have worse health outcomes and worse access to natural environments, yet natural environments may be important to addressing disparities by encouraging PA, improving mental outlook, and reducing psychological stress and thereby increasing life expectancy (Lachowycz \& Jones, 2014). Studies of women in nature have found that fear of natural spaces may add to the under-representation of women in this area of research. In terms of populations with disabilities, there is limited research focusing on individuals with disabilities in nature with most of the research focusing on accessibility of components. Studies have examined the relationship between PA, inactivity, and health among indigenous 
groups and have found results similar to those for other groups with inverse relationships between markers of negative health outcomes with levels of PA (Evans et al., 2018; Macniven et al., 2017; Pelletier et al., 2017).

Additionally, these groups have been shown to have higher rates of obesity (Hedayat et al., 2018; Kumanyika, 2019).

However, this study found no research which included exposure to nature as a variable of interest in these relationships.

\begin{tabular}{|c|c|c|}
\hline & $\begin{array}{l}\text { Trend Among Minorities and Low- } \\
\text { Income Communities }\end{array}$ & Selected Scientific Articles \\
\hline \multicolumn{3}{|l|}{ Access and Awareness } \\
\hline Close Park Proximity & 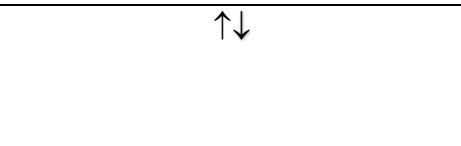 & $\begin{array}{l}\text { (Rigolon, 2017) }^{4} \text {; (Rigolon, 2016) }{ }^{2} \text {; (Taylor et } \\
\text { al., 2007) }{ }^{2} \text {; (Rigolon \& Németh, 2018) } \\
(\text { Jennings \& Gaither, 2015) } \\
2014)^{2} \text {; (Wolch et al., }\end{array}$ \\
\hline Park acreage & $\downarrow$ & $\begin{array}{l}\text { (Rigolon, 2017) }{ }^{4} ;(\text { Rigolon, 2016) })^{2} ;(\text { Weiss et } \\
\text { al., 2011) }{ }^{4} ;(\text { Rigolon \& Németh, 2018) } \\
(\text { Cohen et al., 2016) } \\
\text { Mavoa, et al., 2014) }\end{array}$ \\
\hline Number of parks & $\uparrow \downarrow$ & $\begin{array}{l}\text { (Rigolon, 2016) }{ }^{2} \text {; (Weiss et al., 2011) } \\
\text { (Vaughan et al., 2013) } \\
\text { (Abercrombie et (Moore et al., 2008) } 2008)^{4} \text {; } \text {; (Gordon-Larsen } \\
\text { et al., 2006) }\end{array}$ \\
\hline Low/no cost & $\downarrow$ & $(\text { Moore et al., 2008) })^{4}$ (Dahmann et al., 2010) \\
\hline $\begin{array}{l}\text { Number of facilities/programs within } \\
\text { park }\end{array}$ & $\uparrow \downarrow$ & $\begin{array}{l}\left(\text { Kamel et al., 2014) }{ }^{4} ;(\text { Cohen et al., 2013 })^{4} ;\right. \\
\text { (Moore et al., 2008) })^{4} \text { (Dahmann et al., 2010) }\end{array}$ \\
\hline Sense of belonging & $\downarrow$ & $\begin{array}{l}\text { (Finney, 2014) })^{2} \text {; (Byrne, 2012) }{ }^{4} \text {; (Das et al., } \\
\text { 2017) }\end{array}$ \\
\hline Awareness of national parks & $\downarrow$ & $(\text { Xiao et al., 2018) })^{4}$ (Johnson et al., 2007) \\
\hline \multicolumn{3}{|l|}{ Quality of Space } \\
\hline Poor quality amenities & $\uparrow \downarrow$ & 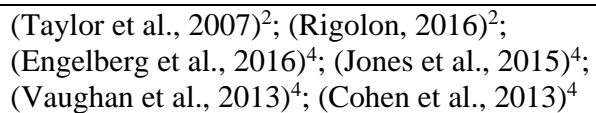 \\
\hline Safety/maintenance & $\downarrow$ & $\begin{array}{l}\text { (Moore et al., 2008) })^{4} \text { (Rigolon, 2017) } \\
\text { (Rigolon, 2016) }{ }^{2} \text {; (Kamel et al., 2014); (Das et } \\
\text { al., 2017) }{ }^{4}\end{array}$ \\
\hline Safety from crime & $\downarrow$ & $\begin{array}{l}(\text { Weiss et al., 2011) })^{4} \text {; Kamel et al., 2014); } \\
(\text { Wolch et al., 2014) }\end{array}$ \\
\hline Parks perceived as less safe & $\uparrow$ & 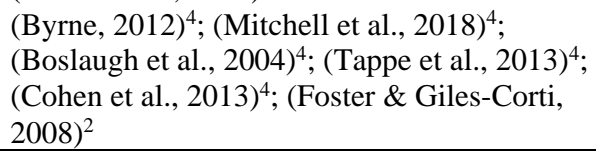 \\
\hline \multicolumn{3}{|l|}{ Funding } \\
\hline For natural space/parks & $\downarrow$ & (Henderson \& Fry, 2011) \\
\hline $\begin{array}{l}{ }^{1} \text { Experimental study design } \\
{ }^{2} \text { Literature Review or meta-analysis } \\
{ }^{3} \text { Quasi experimental study design } \\
{ }^{4} \text { Non-experimental/observational stuc } \\
\uparrow \text { Increased } \\
\downarrow \text { Decreased } \\
\uparrow \downarrow \text { Conflicting results }\end{array}$ & design & \\
\hline
\end{tabular}

across the lifespan. These benefits stem from both the exposure to nature itself and engaging in PA and when combined have the potential for exponential return. However, access to nature components for PA is not universal and therefore presents an opportunity for public health to intervene. 


\section{Gaps in knowledge}

This review focused primarily on positive associations between exposure to nature-based components and PA and health outcomes, in support of the research agenda put forth by Frumkin et al. (2017). We noted several limitations of the current state of literature including the lack of highquality study designs. Most of the research was crosssectional with few cohort or experimental study designs such as randomized clinical trials. Some limitations that contributed to the paucity of well-designed research is the lack of a consistent definition and measurement of nature exposure and standardized terminology, including the type (e.g., green, blue, brown), quality, and duration of exposure as well as the difficulty of designing a control condition. Further, many studies only explore acute health responses rather than long-term outcomes. As suggested by Thompson Coon et al. (2011, p. 1761), "Large, well designed, longer term trials in populations who might benefit most from the potential advantages of outdoor exercise are needed to fully elucidate the effects on mental and physical well-being." A few such studies are being undertaken. Examples from the US include a cluster randomized trial (South et al., 2018), two randomized controlled trials of park prescriptions (Müller-

Riemenschneider et al., 2020; Razani et al., 2018), and two natural experiments that have recently received funding from the National Institutes of Health (Pearson et al., 2020; U.S. National Library of Medicine, 2020). Yet, given the potential of nature and nature-based PA for treatment and management of health and well-being, much more work is needed which will require greater research funding at the federal level. Another gap in knowledge exists in research focusing on access to high quality nature-based components among non-white, marginalized, and vulnerable populations. While much of the current research has focused on the perceptions of these populations related to access, there is a need for more research on why these perceptions exist in the first place in terms of history of segregation of shared public space and the impact on the current state of "who belongs" in these spaces.

Other limitations are associated with both the paucity and inconsistency of objective measures of health outcomes. Thanks to technological advances such as mobile EEG (Aspinall et al., 2015) and the development of field-friendly methods for collecting blood samples (e.g., dried blood spots; McDade et al., 2007) and saliva (Adam \& Kumari, 2009), the use of biomarkers is increasing and available to researchers outside of traditional biomedical settings. However, the diversity of biomarkers available makes it difficult to compare outcomes across studies (Kondo et al., 2018; Twohig-Bennett \& Jones, 2018). It is hoped that as the field advances there will be increased harmonization in the selection of biomarkers as their use expands. Finally, different stakeholders may desire information about different types of objective measures. For example, health insurance companies may value information about the number of visits to providers, but physicians may value data on the changes in a biomarker related to a specific disease. Thus, knowing the audience will be important as the field moves forward.

It is also important to note that while research provides support for the health benefits of exposure to nature-based components and PA, specific populations may not potentially benefit equally. This could be especially true for populations suffering from certain mental health illnesses, such as post-traumatic stress disorder (PTSD), stemming from traumatic events that occurred in outdoor environments. More research is needed to understand the effect of nature exposure on these populations and potentially how best to facilitate that exposure to create positive experiences.

\section{Recommendations and action steps for increasing access to nature-based PA}

The 2013 APHA policy statement explicitly states that increasing exposure to nature should be considered a public health issue regardless of any connections with PA and provides action steps that should be taken to increase access to nature for all populations (Chawla \& Litt, 2013). The literature presented here provides support for increasing access and exposure to nature to increase naturebased PA to further increase health benefits over other forms of PA (indoor or active outdoor). However, as indicated by Frumkin et al., (2017), it is important to note that although the current evidence sufficiently supports action, it is clear that more large-scale biomedical research is needed. This will require increased support from major funding agencies such as the National Institutes of Health to fully examine the type and dose of nature exposure on clinical health outcomes in order to assess the potential of nature and nature-based PA as a prevention and/or treatment strategy.

\section{Improving Access to Nature for All Populations}

City and town governments, planners, parks and recreation agencies, and private organizations should all prioritize access to nature regardless of urbanicity, rurality, and socioeconomic level. Improving access to natural settings can provide increased opportunity for PA for populations at risk of inactivity. Several tools exist to assess access, maintenance, and to facilitate park use such as the Electronic Community Park Audit Tool, and others (eCPAT/ParkAdvisor; Bedimo-Rung et al., 2006; Besenyi et al., 2016; Besenyi et al., 2018; Gustat et al., 2020).

Currently, access to green space is inequitably distributed (Dai, 2011). A recent report suggests that parks that serve people of color are half the size and five times as crowded as parks that serve majority white populations and parks that serve low-income households are one quarter the size and four times as crowded as parks serving highincome households (Trust for Public Land, 2020). Data on the relationships between health outcomes, access to green space, and the presence of socioeconomic and racial/ethnic disparities, while at times conflicting, suggest that living near high quality green spaces improves health and well- 
being (Browning \& Rigolon, 2018). Structural and individual characteristics may influence greenspace use among low-income households (Cronin-de-Chavez et al., 2019). Park and green space use is influenced by culture and ethnicity (Egerer et al., 2019; Rishbeth et al., 2019; Seaman et al., 2010; Sefcik et al., 2019; Zenk et al., 2020). Among other things, concerns for safety, presence of trash, poor amenities, lack of knowledge of where to go, or inability to travel to a space may limit people's use of parks and green space. However, improving nature-based components in low-income areas also comes with inherent risk, such as the issue of green gentrification whereby these improved areas begin to attract wealthier residents that subsequently leads to displacement of low-income households (Anguelovski et al., 2019). Rigolon and Christensen (2019) have identified several strategies for various public sectors to reduce these negative impacts including engaging local community organizations and the people they serve to educate local government officials and policymakers about potential threats and challenges of creating new parks and green space to nearby neighborhoods. Adhering to the principles of community engaged programming and research presents another strategy to involve residents at the local level (Dick, 2017). While disparities in the presence of parks, vegetation, and high quality green spaces are associated with poorer health outcomes, more rigorous studies of how people want to use the spaces are needed to ensure that the spaces provided meet the needs of the people they are intended to serve while decreasing the potential negative impact associated with green gentrification (Mears \& Brindley, 2019). One way to develop equitable advocacy is to include local trusted community champions representing underresourced populations when completing community systems planning.

\section{Education on Nature and Nature-Based PA}

While the benefits of PA are fairly well known to the public, the added health benefits of time spent in nature and nature-based PA are not. Therefore, public health and health care professionals should educate and promote nature-based PA. Currently, there is a growing movement whereby health care providers prescribe nature-based PA to patients in a similar fashion to medication, however, these health care providers seldom receive training or insurance reimbursement to write these prescriptions or counsel patients on nature-based PA (James et al., 2019; Swinburn et al., 1997; Van den Berg, 2017). Therefore, incorporating nature-based PA and related evidence into professional curricula for public health and health care professionals and providing park prescription program training is needed to increase education and support (Besenyi et al., 2020). Additionally, health insurance companies should provide appropriate reimbursement to health care providers who take the time to counsel patients on nature-based PA.

Examples of successfully promoting nature-based PA exist such as "walk and talk therapy" where patient and therapist take a walk in nature while talking during a therapeutic session (van den Berg \& Beute, 2021). To promote nature-based PA during the winter months throughout Canada, colorfully lighted public spaces, art structures, and neighborhoods as well as outdoor markets promote walking while public "warming huts" make various nature-based activities more comfortable (Dobrota $\&$ Armour, 2020). Another strategy that several local public health departments have adopted is to dedicate staff to promoting nature-based PA as was done in Mesa County, Colorado where a full-time permanent Trail Consultant position was created (Mesa County Public Health, 2021).

\section{Increased Access to Community Nature-Based Components}

Creating nature-based components in communities, such as gardens, pocket parks, parks, access to water, and trails, can increase opportunities for nature-based PA, especially in urban areas. This can be done in communities to positively change perceptions of neighborhood safety that can promote walking, biking, and other forms of PA. For all youths, nature provides settings that can promote PA through play by increasing non-competitive and unstructured forms of play. For these reasons, it is important that school grounds be considered for strategies to improve child health (Dyment \& Bell, 2008; Herrington $\&$ Brussoni, 2015). These can include school gardens and natural landscaping in schoolyards for outdoor recess. Audit tools such as the Play Space Audit Tool exist to assist communities in assessing their play spaces (Gustat et al., 2020). Whenever possible, a research and evaluation component should be included with such interventions to provide much needed data on long-term impacts on PA and health outcomes.

\section{Improving Safe Active Transportation}

Active transportation refers to forms of PA that can be used to get from one point to another (e.g., parks and green space) such as walking and biking. Active transportation has been shown to be associated with higher overall levels of PA (Smith et al., 2017). Programs such as Safe Routes to School (Safe Routes Partnership, 2021b), Safe Routes to Play (GP RED, 2018), and Safe Routes to Parks (Safe Routes Partnership, 2021a) are examples of efforts to promote active transportation in communities. Naturebased strategies such as building and networking complete streets, trails, and greenways can be employed to connect access to nature-based components.

\section{Conclusion}

The physical and mental health benefits of nature and PA are well established, however more research is needed to fully understand the relationship between exposure to nature-based components and PA. This scoping review emphasizes that there is ample evidence to support that action steps be taken to increase access to nature-based PA to provide a greater health benefit through the combined effects of exposure to nature-based components for PA.

\section{Author Contributions}


Conceptualization, R.W.C., G.M.B., J.G., T.H.H., T.L.P., and C.L.S.; Investigation, R.W.C., G.M.B., J.G., T.H.H., T.L.P., and C.L.S.; Project Administration, R.W.C.; Writing - Original draft, R.W.C., G.M.B., J.G., T.H.H., T.L.P., and C.L.S.; Writing - Review \& editing, R.W.C., G.M.B., J.G., T.H.H., T.L.P., and C.L.S.

\section{Acknowledgements}

Physical Activity Policy Research and Evaluation Network (PAPREN) is a thematic research network of the Prevention Research Centers program of the US Centers for Disease Control and Prevention, Division of Nutrition, Physical Activity, and Obesity (https://www.papren.org). PAPREN seeks to advance the evidence base supporting physical activity policy and translation of evidence into practice at local, state, and national levels. Salary support for Teresa H. Horton was provided by a gift from The Negaunee Foundation to the Laboratory for Human Biology Research at Northwestern University. Salary support for Teresa L. Penbrooke was provided by GP RED and GreenPlay LLC.

\section{Conflict of interest statement:}

We have no conflicts of interest to disclose.

\section{Creative Commons License:}

This work is licensed under a Creative Commons Attribution-Noncommercial 4.0 International License (CC BY-NC 4.0).

\section{Correspondence should be addressed to}

Richard W. Christiana, PhD, Associate Professor 1179 State Farm Road

Beaver College of Health Sciences

Appalachian State University, Boone, NC 28608 828-262-6335

christianarw@appstate.edu

Richard W. Christiana 0000-0002-9753-4695

Gina M. Besenyi 0000-0002-4538-9510

Jeanette Gustat 0000-0001-5597-1611

Teresa H. Horton 0000-0002-3734-8065

Teresa L. Penbrooke 0000-0002-4705-7472

Courtney L. Schultz 0000-0002-3232-0872

\section{References}

Adam, E. K., \& Kumari, M. (2009). Assessing salivary cortisol in large-scale, epidemiological research. Psychoneuroendocrinology, 34(10), 1423-1436.

Anguelovski, I., Connolly, J. T., Pearsall, H., Shokry, G., Checker, M., Maantay, J., Gould, K., Lewis, T., Maroko, A., \& Roberts, J. T. (2019). Opinion: Why green "climate gentrification" threatens poor and vulnerable populations. Proceedings of the National Academy of Sciences of the United States of America, 116(52), 26139-26143. https://doi.org/https://doi.org/10.1073/pnas.1920490117

Aspinall, P., Mavros, P., Coyne, R., \& Roe, J. (2015). The urban brain: analysing outdoor physical activity with mobile EEG. British Journal of Sports Medicine, 49(4), 272-276.

Astell-Burt, T., Feng, X., \& Kolt, G. S. (2014). Is neighborhood green space associated with a lower risk of type 2 diabetes? Evidence from 267,072 Australians. Diabetes Care, 37(1), 197-201. https://doi.org/10.2337/dc13-1325

Bedimo-Rung, A. L., Gustat, J., Tompkins, B. J., Rice, J., \& Thomson, J. (2006). Developement of a direct observation instrument to measure environmental characteristics of parks for physical activity. Journal of Physical Activity \& Health, 3(S1), S176-S189. https://doi.org/10.1123/jpah.3.s1.s176

Beery, T., Jönsson, K., \& Elmberg, J. (2015). From environmental connectedness to sustainable futures: Topophilia and human affiliation with nature. Sustainability, 7(7), 8837-8854.

Besenyi, G. M., Diehl, P., Schooley, B., Turner-McGrievy, B. M., Wilcox, S., Wilhelm Stanis, S. A., \& Kaczynski, A. T. (2016). Development and testing of mobile technology for community park improvements: Validity and reliability of the eCPAT application with youth. Translational Behavioral Medicine, 6(4), 519-532.

Besenyi, G. M., Hayashi, E. B., \& Christiana, R. W. (2020). Prescribing physical activity in parks and nature: Health care provider insights on park prescription programs. Journal of Physical Activity \& Health, 17(10), 958-967. https://doi.org/10.1123/jpah.2019-0479

Besenyi, G. M., Schooley, B., Turner-McGrievy, G. M., Wilcox, S., Wilhelm Stanis, S. A., \& Kaczynski, A. T. (2018). The Electronic Community Park Audit Tool (eCPAT): Exploring the use of mobile technology for youth empowerment and advocacy for healthy community policy, systems, and environmental change. Frontiers in Public Health, 6, 332.

Beyer, K. M., Szabo, A., Hoormann, K., \& Stolley, M. (2018). Time spent outdoors, activity levels, and chronic disease among American adults. Journal of Behavioral Medicine, 41(4), 494-503. 
Bowler, D. E., Buyung-Ali, L. M., Knight, T. M., \& Pullin, A. S. (2010). A systematic review of evidence for the added benefits to health of exposure to natural environments. BMC Public Health, 10(1), 456.

Bratman, G. N., Anderson, C. B., Berman, M. G., Cochran, B., de Vries, S., Flanders, J., Folke, C., Frumkin, H., Gross, J. J., Hartig, T., Kahn, P. H., Jr., Kuo, M., Lawler, J. J., Levin, P. S., Lindahl, T., Meyer-Lindenberg, A., Mitchell, R., Ouyang, Z., Roe, J., Scarlett, L., Smith, J. R., van den Bosch, M., Wheeler, B. W., White, M. P., Zheng, H., \& Daily, G. C. (2019). Nature and mental health: An ecosystem service perspective. Science Advances, 5(7), eaax0903. https://doi.org/10.1126/sciadv.aax0903

Browning, M., \& Rigolon, A. (2018). Do income, race and ethnicity, and sprawl influence the greenspace-human health link in city-level analyses? Findings from 496 cities in the United States. International Journal of Environmental Research and Public Health, 15(7), 1-22. https://doi.org/10.3390/ijerph15071541

Bullard, R. D. (2021). Dr. Robert Bullard: Father of Environmental Justice. Retrieved September 10 from https://drrobertbullard.com/

Centers for Disease Control and Prevention. (2009). Healthy places terminology. Centers for Disease Control and Prevention, USA. Retrieved 5/23/2019 from http://www.cdc.gov/healthyplaces/terminology.htm\#d

Chawla, L., \& Litt, J. S. (2013). Improving Health and Wellness through Access to Nature (Policy Number 20137). https://www.apha.org/policies-and-advocacy/public-health-policy-statements/policydatabase/2014/07/08/09/18/improving-health-and-wellness-through-access-to-nature

Cohen, D. A., Ashwood, J. S., Scott, M. M., Overton, A., Evenson, K. R., Staten, L. K., Porter, D., McKenzie, T. L., \& Catellier, D. (2006). Public parks and physical activity among adolescent girls. Pediatrics, 118(5), 1381-1389.

Coombes, E., Jones, A. P., \& Hillsdon, M. (2010). The relationship of physical activity and overweight to objectively measured green space accessibility and use. Social Science and Medicine, 70(6), 816-822. https://doi.org/DOI 10.1016/j.socscimed.2009.11.020

Cronin-de-Chavez, A., Islam, S., \& McEachan, R. R. C. (2019). Not a level playing field: A qualitative study exploring structural, community and individual determinants of greenspace use amongst low-income multi-ethnic families. Health \& Place, 56, 118-126. https://doi.org/https://doi.org/10.1016/j.healthplace.2019.01.018

Dadvand, P., Rivas, I., Basagana, X., Alvarez-Pedrerol, M., Su, J., De Castro Pascual, M., Amato, F., Jerret, M., Querol, X., Sunyer, J., \& Nieuwenhuijsen, M. J. (2015). The association between greenness and traffic-related air pollution at schools. Science of the Total Environment, 523, 59-63. https://doi.org/10.1016/j.scitotenv.2015.03.103

Dai, D. (2011). Racial/ethnic and socioeconomic disparities in urban green space accessibility: Where to intervene. Landscape and Urban Planning, 102, 234-244. https://doi.org/http://www.sciencedirect.com/science/article/pii/S0169204611001952

De Vries, S. (2019). Comparison of the Health Benefits of Different Types of Nature. JAMA network open, 2(7), e198215e198215.

Detweiler, M. B., Carman, J., \& J.G., M.-D. (2018). Nature-based treatments as an adjunctive therapy for anxiety among elders. In M. van den Bosch \& W. Bird (Eds.), Oxford Textbook of Nature and Public Health (pp. 177-181). Oxford University Press.

Dick, D. M. (2017). Rethinking the way we do research: The benefits of community-engaged, citizen science approaches and nontraditional collaborators. Alcoholism, Clinical and Experimental Research, 41(11), 1849-1856.

Dobrota, A., \& Armour, G. (2020). Winter placemaking during a pandemic: Six ideas from around Canada. Project for Public Spaces. Retrieved September 8 from https://www.pps.org/article/winter-placemaking-during-a-pandemic-six-ideasfrom-around-canada

Donovan, G. H., Butry, D. T., Michael, Y. L., Prestemon, J. P., Liebhold, A. M., Gatziolis, D., \& Mao, M. Y. (2013). The Relationship Between Trees and Human Health Evidence from the Spread of the Emerald Ash Borer. American Journal of Preventive Medicine, 44(2), 139-145. https://doi.org/DOI 10.1016/j.amepre.2012.09.066

Du, Y., Liu, B., Sun, Y., Snetselaar, L. G., Wallace, R. B., \& Bao, W. (2019). Trends in adherence to the Physical Activity Guidelines for Americans for aerobic activity and time spent on sedentary behavior among US adults, 2007 to 2016. JAMA network open, 2(7), e197597-e197597.

Dyment, J. E., \& Bell, A. C. (2008). Grounds for movement: green school grounds as sites for promoting physical activity. Health Education Research, 23(6), 952-962. 
Egerer, M., Ordóñez, C., Lin, B. B., \& Kendal, D. (2019). Multicultural gardeners and park users benefit from and attach diverse values to urban nature spaces. Urban Forestry \& Urban Greening, 46, 126445. https://doi.org/https://doi.org/10.1016/j.ufug.2019.126445

Evans, J. R., Wilson, R., Coleman, C., Man, W. Y. N., \& Olds, T. (2018). Physical activity among indigenous Australian children and youth in remote and non-remote areas. Social science \& medicine (1982), 206, 93-99. https://doi.org/10.1016/j.socscimed.2018.04.018

Finney, C. (2014). Black faces, white spaces: Reimagining the relationship of African Americans to the great outdoors. The University of North Carolina Press.

Flowers, E. P., Freeman, P., \& Gladwell, V. F. (2016). A cross-sectional study examining predictors of visit frequency to local green space and the impact this has on physical activity levels. BMC Public Health, 16, 420. https://doi.org/10.1186/s12889-016-3050-9

Fraser, M., Munoz, S.-A., \& MacRury, S. (2019). What Motivates Participants to Adhere to Green Exercise? International Journal of Environmental Research and Public Health, 16(10), 1832.

Frumkin, H., Bratman, G. N., Breslow, S. J., Cochran, B., Kahn, P. H., Jr., Lawler, J. J., Levin, P. S., Tandon, P. S., Varanasi, U., Wolf, K. L., \& Wood, S. A. (2017). Nature Contact and Human Health: A Research Agenda. Environmental Health Perspectives, 125(7), 075001-075001. https://doi.org/10.1289/EHP1663

GP RED. (2018). Safe Routes to Play (SRTP). https://www.gpred.org/initiatives/safe-routes-toplay/\#: :text=Safe\%20Routes\%20to\%20Play\%20(SRTP)\%20is\%20a\%20child\%2Dcentered,for\%20children\%20and\% 20their\%20families

Gustat, J., Anderson, C. E., \& Slater, S. J. (2020). Development and testing of a brief play space audit tool. Journal of Physical Activity \& Health, 17(1), 13-20.

Hagerhall, C. (2018). Responses to nautre from populations of varied cultural background. In M. van den Bosch \& W. Bird (Eds.), Oxford Textbook of Nature and Public Health (pp. 189-192). Oxford University Press.

Hartig, T., Mitchell, R., Vries, S. d., \& Frumkin, H. (2014). Nature and Health. Annual Review of Public Health, 35(1), $207-228$. https://doi.org/10.1146/annurev-publhealth-032013-182443

Hedayat, L. M. A., Murchison, C. C., \& Foulds, H. J. A. (2018). A systematic review and meta-analysis of cardiorespiratory fitness among Indigenous populations in North America and circumpolar Inuit populations. Preventive Medicine, 109, 71-81. https://doi.org/10.1016/j.ypmed.2018.01.007

Herrington, S., \& Brussoni, M. (2015). Beyond physical activity: The importance of play and nature-based play spaces for children's health and development. Current obesity reports, 4(4), 477-483.

Hofferth, S. L., \& Sandberg, J. F. (2001). How American children spend their time. Journal of Marriage and Family, 63(2), 295308.

James, J. J., Christiana, R. W., \& Battista, R. A. (2019). A historical and critical analysis of park prescriptions. Journal of Leisure Research, 50(4), 311-329. https://doi.org/10.1080/00222216.2019.1617647

Janssen, I., \& Rosu, A. (2015). Undeveloped green space and free-time physical activity in 11 to 13 -year-old children. International Journal of Behavioral Nutrition and Physical Activity, 12(1), 26.

Johnson, D. L., Ambrose, S. H., Bassett, T. J., Bowen, M. L., Crummey, D. E., Isaacson, J. S., Johnson, D. N., Lamb, P., Saul, M., \& Winter-Nelson, A. E. (1997). Meanings of environmental terms. Journal of Environmental Quality, 26(3), 581589.

Joye, Y., \& De Block, A. (2011). 'Nature and I are Two': A Critical Examination of the Biophilia Hypothesis. Environmental Values, 20(2), 189-215.

Juster, F. T., Ono, H., \& Stafford, F. P. (2004). Changing times of American youth: 1981-2003. Institute for Social Research, University of Michigan, Ann Arbor, Michigan, 1-15.

Kaplan, R., \& Kaplan, S. (1989). The experience of nature: A psychological perspective. CUP Archive. 
Katzmarzyk, P. T., Denstel, K. D., Beals, K., Carlson, J., Crouter, S. E., McKenzie, T. L., Pate, R. R., Sisson, S. B., Staiano, A. E., Stanish, H., Ward, D. S., Whitt-Glover, M., \& Wright, C. (2018). Results from the United States 2018 Report Card on Physical Activity for Children and Youth. 15(s2), S422. https://doi.org/10.1123/jpah.2018-0476

Kellert, S. R., \& Case, D. J. (2018). The Nature of Americans. D.J. Case and Associates. Retrieved 1/31/2018 from https://natureofamericans.org/

Kellert, S. R., \& Wilson, E. O. (1995). The Biophilia Hypothesis, 1993 (Vol. 15). Island Press. https://doi.org/10.1177/027046769501500125

Kelly, C. (2018). 'I Need the Sea and the Sea Needs Me': Symbiotic coastal policy narratives for human wellbeing and sustainability in the UK. Marine Policy, 97, 223-231.

Klepeis, N. E., Nelson, W. C., Ott, W. R., Robinson, J. P., Tsang, A. M., Switzer, P., Behar, J. V., Hern, S. C., \& Engelmann, W. H. (2001). The National Human Activity Pattern Survey (NHAPS): a resource for assessing exposure to environmental pollutants. Journal of Exposure Science \& Environmental Epidemiology, 11(3), 231-252.

Klompmaker, J. O., Hoek, G., Bloemsma, L. D., Gehring, U., Strak, M., Wijga, A. H., Brink, C. v. d., Brunekreef, B., Lebret, E., \& Janssen, N. A. (2018). Green space definition affects associations of green space with overweight and physical activity. Environmental Research, 160(1), 531-540. https://doi.org/https://doi.org/10.1016/j.envres.2017.10.027

Knez, I., Ode Sang, Å., Gunnarsson, B., \& Hedblom, M. (2018). Wellbeing in urban greenery: the role of naturalness and place identity. Frontiers in Psychology, 9, 491.

Kondo, M. C., Fluehr, J. M., McKeon, T., \& Branas, C. C. (2018). Urban Green Space and Its Impact on Human Health. International Journal of Environmental Research and Public Health, 15(3). https://doi.org/10.3390/ijerph15030445

Koohsari, M. J., Karakiewicz, J. A., \& Kaczynski, A. T. (2013). Public open space and walking: the role of proximity, perceptual qualities of the surrounding built environment, and street configuration. Environment and Behavior, 45(6), 706-736.

Kumanyika, S. K. (2019). Unraveling common threads in obesity risk among racial/ethnic minority and migrant populations. Public Health, 172, 125-134. https://doi.org/10.1016/i.puhe.2019.04.010

Kuo, M. (2015). How might contact with nature promote human health? Promising mechanisms and a possible central pathway. Front Psychol, 6, 1093. https://doi.org/10.3389/fpsyg.2015.01093

Lachowycz, K., \& Jones, A. P. (2011). Greenspace and obesity: a systematic review of the evidence. Obesity reviews : an official journal of the International Association for the Study of Obesity, 12(5), e183-189. https://doi.org/10.1111/j.1467789X.2010.00827.x; 10.1111/j.1467-789X.2010.00827.x

Lachowycz, K., \& Jones, A. P. (2013). Towards a better understanding of the relationship between greenspace and health: Development of a theoretical framework. Landscape and Urban Planning, 118, 62-69.

Lachowycz, K., \& Jones, A. P. (2014). Does walking explain associations between access to greenspace and lower mortality? Social Science and Medicine, 107, 9-17. https://doi.org/https://doi.org/10.1016/j.socscimed.2014.02.023

Layton, R. D. (2018). Parks as a health treatment: Measuring the dosage Council of Educators in Landscape Architecture, Blacksburg, VA.

Lee, K. J., \& Scott, D. (2016). Bourdieu and African Americans' Park Visitation: The Case of Cedar Hill State Park in Texas. Leisure Sciences, 38(5), 424-440. https://doi.org/https://doi.org/10.1080/01490400.2015.1127188

Li, F., Fisher, K. J., Brownson, R. C., \& Bosworth, M. (2005). Multilevel modelling of built environment characteristics related to neighbourhood walking activity in older adults. Journal of Epidemiology and Community Health, 59(7), 558-564. https://doi.org/10.1136/jech.2004.028399

Livesley, S. J., McPherson, G. M., \& Calfapietra, C. (2016). The Urban Forest and Ecosystem Services: Impacts on Urban Water, Heat, and Pollution Cycles at the Tree, Street, and City Scale. J Environ Qual, 45(1), 119-124. https://doi.org/10.2134/jeq2015.11.0567

Livingston, G. (2019). Americans 60 and older are spending more time in front of their screens than a decade ago. Pew Research Center. Retrieved 2/10/2020 from https://pewrsr.ch/2ZlCbpn 
Locke, D. H., Hall, B., Grove, J. M., Pickett, S. T. A., Ogden, L. A., Aoki, C., Boone, C. G., \& O’Neil-Dunne, J. P. M. (2021). Residential housing segregation and urban tree canopy in 37 US cities. NPJ Urban Sustainability, 1(15), 1-9. https://doi.org/https://doi.org/10.1038/s42949-021-00022-0

Lowry, C. A., Smith, D. G., Siebler, P. H., Schmidt, D., Stamper, C. E., Hassell, J. E., Jr., Yamashita, P. S., Fox, J. H., Reber, S. O., Brenner, L. A., Hoisington, A. J., Postolache, T. T., Kinney, K. A., Marciani, D., Hernandez, M., Hemmings, S. M., Malan-Muller, S., Wright, K. P., Knight, R., Raison, C. L., \& Rook, G. A. (2016). The Microbiota, Immunoregulation, and Mental Health: Implications for Public Health. Curr Environ Health Rep, 3(3), 270-286. https://doi.org/10.1007/s40572-016-0100-5

Macniven, R., Hearn, S., Grunseit, A., Richards, J., Nutbeam, D., \& Bauman, A. (2017). Correlates of physical activity among Australian Indigenous and non-Indigenous adolescents. Australian and New Zealand Journal of Public Health, 41(2), 187-192. https://doi.org/10.1111/1753-6405.12609

Matz, C. J., Stieb, D. M., Davis, K., Egyed, M., Rose, A., Chou, B., \& Brion, O. (2014). Effects of age, season, gender and urban-rural status on time-activity: Canadian Human Activity Pattern Survey 2 (CHAPS 2). International Journal of Environmental Research and Public Health, 11(2), 2108-2124.

McDade, T. W., Williams, S., \& Snodgrass, J. J. (2007). What a drop can do: Drid blood spots as a minimally invasive method for integrating biomarkers into population-based research. Demography, 44(4), 899-925. https://doi.org/https://link.springer.com/article/10.1353/dem.2007.0038

McDonald, R. I., Beatley, T., \& Elmqvist, T. (2018). The green soul of the concrete jungle: the urban century, the urban psychological penalty, and the role of nature [journal article]. Sustainable Earth, 1(1), 3. https://doi.org/10.1186/s42055-018-0002-5

McIsaac, G. F., \& Brün, M. (1999). Natural environment and human culture: defining terms and understanding worldviews. Journal of Environmental Quality, 28(1), 1-10.

Mears, M., \& Brindley, P. (2019). Measuring Urban Greenspace Distribution Equity: The Importance of Appropriate Methodological Approaches. ISPRS International Journal of Geo-Information, 8(6), 286. https://www.mdpi.com/2220$\underline{9964 / 8 / 6 / 286}$

Mesa County Public Health. (2021). Mesa County Public Health. Retrieved September 10 from https://health.mesacounty.us/

Müller-Riemenschneider, F., Petrunoff, N., Yao, J., Ng, A., Sia, A., Ramiah, A., Wong, M., Han, J., Tai, B. C., \& Uijtdewilligen, L. (2020). Effectiveness of prescribing physical activity in parks to improve health and wellbeing - the park prescription randomized controlled trial. International Journal of Behavioral Nutrition and Physical Activity, 17(42), 114. https://doi.org/10.1186/s12966-020-00941-8

Nazif-Munoz, J. I., Cedeno Laurent, J. G., Browning, M., Spengler, J., \& Olvera Álvarez, H. A. (2020). Green, brown, and gray: Associations between different measurements of land patterns and depression among nursing students in El Paso, Texas. International Journal of Environmental Research and Public Health, 17(21), 8146.

Oh, B., Lee, K. J., Zaslawski, C., Yeung, A., Rosenthal, D., Larkey, L., \& Back, M. (2017). Health and well-being benefits of spending time in forests: systematic review. Environ Health Prev Med, 22(1), 71. https://doi.org/10.1186/s12199-017$\underline{0677-9}$

Olafsdottir, G., Cloke, P., \& Vögele, C. (2017). Place, green exercise and stress: An exploration of lived experience and restorative effects. Health \& Place, 46, 358-365.

Outdoor Foundation. (2021). 2021 Outdoor participation trends report. Outdoor Foundation.

Pearson, A. L., Pfeiffer, K. A., Gardiner, J., Horton, T., Buxton, R. T., Hunter, R. F., Breeze, V., \& McDade, T. (2020). Study of active neighborhoods in Detroit (StAND): study protocol for a natural experiment evaluating the health benefits of ecological restoration of parks. BMC Public Health, 20(1), 638. https://doi.org/https://doi.org/10.1186/s12889-020$\underline{08716-3}$

Pelletier, C. A., Smith-Forrester, J., \& Klassen-Ross, T. (2017). A systematic review of physical activity interventions to improve physical fitness and health outcomes among Indigenous adults living in Canada. Preventive Medicine Reports, 8, 242249. https://doi.org/10.1016/j.pmedr.2017.11.002

Piercy, K. L., Troiano, R. P., Ballard, R. M., Carlson, S. A., Fulton, J. E., Galuska, D. A., George, S. M., \& Olson, R. D. (2018). The Physical Activity Guidelines for Americans. JAMA, 320(19), 2020-2028. https://doi.org/10.1001/jama.2018.14854 
Pinter-Wollman, N., Jelic, A., \& Wells, N. M. (2018). The impact of the built environment on health behaviours and disease transmission in social systems. Philosophical Transactions of the Royal Society B-Biological Sciences, 373(1753). https://royalsocietypublishing.org/doi/full/10.1098/rstb.2017.0245

Prescott, S. L., \& Logan, A. C. (2016). Transforming Life: A Broad View of the Developmental Origins of Health and Disease Concept from an Ecological Justice Perspective. International Journal of Environmental Research and Public Health, 13(11). https://doi.org/10.3390/ijerph13111075

Pretty, J., Griffin, M., Sellens, M., \& Pretty, C. (2003). Green Exercise: Complementary Roles of Nature, Exercise and Diet in Physical and Emotional Well-Being and Implications for Public Health Policy. University of Essex.

Pretty, J., Peacock, J., Sellens, M., \& Griffin, M. (2005, Oct). The mental and physical health outcomes of green exercise. International Journal of Environmental Health Research, 15(5), 319-337. http://www.ncbi.nlm.nih.gov/entrez/query.fcgi?cmd=Retrieve \&db=PubMed\&dopt=Citation\&list_uids=16416750

Razani, N., Morshed, S., Kohn, M. A., Wells, N. M., Thompson, D., Alquassari, M., Agodi, A., \& Rutherford, G. W. (2018). Effect of park prescriptions with and without group visits to parks on stress reduction in low-income parents: SHINE randomized trial. PLOS ONE, 13(2), 1-17. https://doi.org/https://doi.org/10.1371/journal.pone.0192921

Ribeiro, A. I., Mitchell, R., Carvalho, M. S., \& de Pina, M. d. F. (2013). Physical activity-friendly neighbourhood among older adults from a medium size urban setting in Southern Europe. Preventive Medicine, 57(5), 664-670.

Rigolon, A., \& Christensen, J. (2019). Greening without gentrification: Learning from parks-related anti-displacement strategies nationwide. Institute of the Environment and Sustainability. https://www.ioes.ucla.edu/wpcontent/uploads/Greening-without-Gentrification-report-2019.pdf

Rishbeth, C., Blachnicka-Ciacek, D., \& Darling, J. (2019). Participation and wellbeing in urban greenspace: 'curating sociability' for refugees and asylum seekers. Geoforum, 106, 125-134. https://doi.org/https://doi.org/10.1016/j.geoforum.2019.07.014

Safe Routes Partnership. (2021a). Safe Routes to Parks. https://www.saferoutespartnership.org/healthycommunities/saferoutestoparks

Safe Routes Partnership. (2021b). Safe Routes to School. https://www.saferoutespartnership.org/safe-routes-school

Sampson, S. D. (2012). The topophilia hypothesis: Ecopsychology meets evolutionary psychology. Ecopsychology: Science, totems, and the technological species, 23-53.

Schiller, J. S., Clarke, T. C., \& Norris, T. (2018). Early release of selected estimates based on data from the January-September 2017 National Health Interview Survey. C. f. D. C. a. P. (USA). https://www.cdc.gov/nchs/data/nhis/earlyrelease/EarlyRelease201803.pdf

Schipperijn, J., Bentsen, P., Troelsen, J., Toftager, M., \& Stigsdotter, U. K. (2013). Associations between physical activity and characteristics of urban green space. Urban Forestry \& Urban Greening, 12(1), 109-116.

Seaman, P. J., Jones, R., \& Ellaway, A. (2010). It's not just about the park, it's about integration too: why people choose to use or not use urban greenspaces. Int J Behav Nutr Phys Act, 7, 78. https://doi.org/10.1186/1479-5868-7-78

Sefcik, J. S., Kondo, M. C., Klusaritz, H., Sarantschin, E., Solomon, S., Roepke, A., South, E. C., \& Jacoby, S. F. (2019). Perceptions of Nature and Access to Green Space in Four Urban Neighborhoods. International Journal of Environmental Research and Public Health, 16(13), 2313. https://www.mdpi.com/1660-4601/16/13/2313

Shanahan, D. F., Astell-Burt, T., Barber, E. A., Brymer, E., Cox, D. T., Dean, J., Depledge, M., Fuller, R. A., Hartig, T., Irvine, K. N., Jones, A., Kikillus, H., Lovell, R., MItchell, R., Niemela, J., Nieuwenhuijsen, M., Pretty, J., Townsend, M., van Heezik, Y., Warber, S., \& Gaston, K. J. (2019). Nature-based interventions for improving health and wellbeing: The purpose, the people and the outcomes. Sports, 7(6), 141-164. https://doi.org/10.3390/sports7060141

Smith, M., Hosking, J., Woodward, A., Witten, A., MacMillan, A., Field, A., Baas, P., \& Mackie, H. (2017). Systematic literature review of built environment effects on physical activity and active transport - an update and new findings on health equity. International Journal of Behavioral Nutrition and Physical Activity, 14(1), 158. https://doi.org/10.1186/s12966-017-0613-9

South, E. C., Hohl, B. C., Kondo, M. C., MacDonald, J. M., \& Branas, C. C. (2018). Effect of greening vacant land on mental health of community-dwelling adults: A cluster randomized trial. JAMA network open, 1(3), e180298. https://doi.org/http://dx.doi.org/10.1001/jamanetworkopen.2018.0298 
Spangler, K. J., \& Caldwell, L. L. (2007). The implications of public policy related to parks, recreation, and public health: a focus on physical activity. Journal of Physical Activity \& Health, 4 Suppl 1(Journal Article), S64-71.

Spencer, C., \& Gee, K. (2009). The roots and branches of environmental psychology. The Psychologist, 22, 180-183. https://thepsychologist.bps.org.uk/volume-22/edition-2/roots-and-branches-environmental-psychology

Stigsdotter, U. K., Palsdottir, A. M., Burls, A., Chermaz, A., Ferrini, F., \& Grahn, P. (2011). Nature-based therapeutic interventions. In Forests, trees, and human health (pp. 309-343). Springer, Dordrecht.

Swinburn, B. A., Walter, L. G., Arroll, B., Tilyard, M. W., \& Russell, D. W. (1997). Green prescriptions: attitudes and perceptions of general practitioners towards prescribing exercise. British Journal of General Practice, 47(422), 567569.

Taylor, L., \& Hochuli, D. F. (2017). Defining greenspace: Multiple uses across multiple disciplines. Landscape and Urban Planning, 158, 25-38.

Thompson Coon, J., Boddy, K., Stein, K., Whear, R., Barton, J., \& Depledge, M. H. (2011). Does participating in physical activity in outdoor natural environments have a greater effect on physical and mental wellbeing than physical activity indoors? A systematic review. Environmental science \& technology, 45(5), 1761-1772.

Trust for Public Land. (2020). The Heat is On: A Trust for Public Land Special Report. Retrieved December 18 from https://www.tpl.org/sites/default/files/The-Heat-is-on_A-Trust-for-Public-Land_special-report.pdf

Tsao, T. M., Tsai, M. J., Hwang, J. S., Cheng, W. F., Wu, C. F., Chou, C. K., \& Su, T. C. (2018). Health effects of a forest environment on natural killer cells in humans: an observational pilot study. Oncotarget, 9(23), 16501-16511. https://doi.org/10.18632/oncotarget.24741

Twohig-Bennett, C., \& Jones, A. (2018). The health benefits of the great outdoors: A systematic review and meta-analysis of greenspace exposure and health outcomes. Environmental Research, 166(1), 628-637. https://doi.org/10.1016/j.envres.2018.06.030

U.S. National Library of Medicine. (2020). Health, Environment and Action in Louisville (HEAL) Green Heart Louisville Project (HEAL). U.S. National Library of Medicine. Retrieved November 15 from https://clinicaltrials.gov/ct2/show/NCT03670524?term=Green+Heart\&cond=Cardiovascular+Diseases\&draw=2\&rank $=1$

Ulrich, R. S., Simons, R. F., Losito, B. D., Fiorito, E., Miles, M. A., \& Zelson, M. (1991). Stress recovery during exposure to natural and urban environments. Journal of Environmental Psychology, 11(3), 201-230.

US Department of Health and Human Services. (2010). Healthy people 2020. https://www.healthypeople.gov/2020

Van den Berg, A. E. (2017). From green space to green prescriptions: Challenges and opportunities for research and practice. Frontiers in Psychology, 8, 268. https://doi.org/10.3389/fpsyg.2017.00268

van den Berg, A. E., \& Beute, F. (2021). Walk it off! The effectiveness of walk and talk coaching in nature for individuals with burnout- and stress-related complaints. Journal of Environmental Psychology, 76(101641), 1-11. https://doi.org/https://doi.org/10.1016/j.jenvp.2021.101641

Völker, S., \& Kistemann, T. (2011). The impact of blue space on human health and well-being-Salutogenetic health effects of inland surface waters: A review. International Journal of Hygiene and Environmental Health, 214(6), 449-460.

World Health Organization. (2016). Urban green spaces and health: A review of evidence. World Health Organization: Copenhagen, Denmark.

Yang, B.-Y., Markevych, I., Heinrich, J., Bowatte, G., Bloom, M. S., Guo, Y., Dharmage, S. C., Jalaludin, B., Knibbs, L. D., \& Morawska, L. (2019). Associations of greenness with diabetes mellitus and glucose-homeostasis markers: The 33 Communities Chinese Health Study. International Journal of Hygiene and Environmental Health, 222(2), 283-290.

Zenk, S. N., Pugach, O., Ragonese-Barnes, M., Odoms-Young, A., Powell, L. M., \& Slater, S., J. (2020). Did playground renovations equitably benefit neighborhoods in Chicago? Journal of Urban Health. https://doi.org/https://doi.org/10.1007/s11524-020-00472-4 